\title{
Competencias que potencian la capacidad gerencial en las personas directivas para el trabajo efectivo con las Juntas Administrativas y de Educación en el sistema educativo costarricense
}

\section{Competences that enhance managerial capacity in managers for effective work with Administrative and Educational Boards in the Costa Rican education system}

\author{
Evelyn Chen-Quesada \\ Académica, División de Educación para el Trabajo \\ Universidad Nacional \\ Heredia, Costa Rica \\ evelyn.chen.quesada@una.cr \\ Warner Ruiz-Chaves \\ Académico, División de Educación para el Trabajo \\ Universidad Nacional \\ Investigador, Centro de Investigaciones en Educación \\ Universidad Estatal a Distancia \\ San José, Costa Rica \\ warner.ruiz@gmail.com
}

Recibido - Received: 28 / 02 / $2020 \quad$ Corregido - Revised: 28 / 04 / 2020 Aceptado - Accepted: 08 / 06/2020

DOI: https://doi.org/10.22458/ie.v22i32.2964

URL: https://revistas.uned.ac.cr/index.php/innovaciones

\begin{abstract}
Resumen: El propósito del artículo es analizar las competencias que potencian la capacidad gerencial de las personas directivas de centros educativos para el trabajo efectivo con las juntas de educación y juntas administrativas en el contexto educativo costarricense. La metodología de la investigación se desarrolló bajo un enfoque cualitativo donde se identificaron las categorías de competencias requeridas mediante grupos focales, entrevistas y encuesta entre directivos que se desempeñan en las Direcciones Regionales de Educación de San José Oeste, San José Central, Alajuela y Heredia del Ministerio de Educación Pública. Como resultado del análisis se determinó que las principales competencias gerenciales que deben tener los directivos de las instituciones educativas para el trabajo efectivo con las juntas son: liderazgo, comunicación, negociación, trabajo en equipo y delegación.
\end{abstract}

Palabras clave: Capacidad, Competencia profesional, Gerencia, Administrador de la educación, Gestión educativa, Articulación educativa

Summary: The purpose of the article is to analyze the competences that enhance the managerial capacity of the directors of education centers for effective work with the boards of education and administrative boards in the Costa Rican educational context. The research methodology was developed under a qualitative approach with a quantitative complement where the categories of required competencies were identified, through focus groups, interviews and a survey among managers who work in the Regional Head Departments of Education of San José West, San José Central, Alajuela and Heredia of the Ministry of Public Education. As a result of the analysis, it was determined that the main managerial competencies that the directors of the educational institutions must have for effective work with the boards are: leadership, communication, negotiation, teamwork and delegation.

Key Words: Capacity, Professional competence, Management, Educational administrator, Educational management, Educational articulation 


\section{INTRODUCCIÓN}

El desarrollo de la capacidad gerencial de las personas directivas tiene un papel fundamental en el trabajo con las juntas de educación y juntas administrativas en el contexto educativo costarricense (en adelante las Juntas). El manuscrito que se presenta, elaborado como parte de las actividades investigativas y de extensión de la División de Educación para el Trabajo (DET) del Centro de Investigación y Docencia en Educación (CIDE) de la Universidad Nacional se propone como objetivo general analizar las competencias que potencian la capacidad gerencial de las personas directivas de centros educativos para el trabajo efectivo con las Juntas. Los objetivos específicos de esta experiencia investigativa son: identificar las competencias requeridas para potenciar la capacidad gerencial del directivo para el trabajo efectivo con las Juntas; determinar la relación de las competencias detectadas de las personas directivas para la atención y solución de problemas de forma colaborativa con las Juntas e identificar los desafíos de las personas directivas de los centros para el trabajo efectivo con las Juntas.

Para lograr lo descrito en el párrafo anterior, es importante señalar que la mejora en los procesos de gestión que realizan las Juntas es clave para el éxito de un centro escolar. Esta es la razón por la que el desarrollo de la capacidad gerencial de las personas Directivas es un tema prioritario para la mejora de la calidad y la concreción de los programas y proyectos que se gestan y ejecutan con las Juntas. De acuerdo con el Decreto 38249 - MEP, denominado Reglamento General de Juntas de Educación y Juntas Administrativas (2014), las Juntas se definen como:

Los organismos auxiliares de la administración pública que constituyen la base para el funcionamiento de los centros educativos públicos, están sometidas a la tutela administrativa del Poder Ejecutivo, por medio del Ministerio de Educación Pública (MEP) como rector del sector educación, con el fin de garantizar que sus actuaciones sean consistentes con la política educativa. (párr. 5-10)

Estos espacios de participación ciudadana, se conforman por cinco personas que trabajan ad honorem, para determinar, gestionar y solventar de conformidad con lo establecido en el Plan Anual de Trabajo (PAT) del centro educativo y los lineamientos técnicos específicos dictados por el MEP. Los miembros de la Junta son propuestos por la persona directiva en coordinación con el personal del centro educativo a la Supervisión de Circuito Escolar, quien verifica los requisitos y ésta última lo tramita ante el Concejo Municipal del Cantón.

La persona directiva (en este estudio se entiende como persona directiva al director o directora del centro educativo) es partícipe de la Junta con derecho a voz pero no a voto. Es una figura importante que brinda el apoyo logístico a la Junta para que se cumplan las necesidades y requerimientos propuestos en el Plan Anual de Trabajo (PAT). Al respecto, en cuanto a antecedentes, en una investigación realizada por Torres y Castro (2012), se determinó que "el director o directora y la junta deben ser mediadores (...), pero entendiendo que la persona directiva juega un papel protagónico como agente social que asume el tema de participación como alternativa pedagógica para generar procesos de transformación" (p. 111).

Del mismo modo, el rol de la persona directiva es fundamental para el logro de los proyectos del centro, por lo que toda persona que aspire a llegar al puesto, no solo debe tener la preparación académica, sino la capacidad gerencial para ese trabajo con la Junta. En este sentido, Patrón-Cortés (2016) determinó que existen diferencias entre conocimientos y competencias, pues aunque los directores tienen la preparación que les permite conocer aspectos relacionados con la administración educativa, se les dificulta el aplicarlos. Los directores manejan más las competencias requeridas para el logro de resultados y la unidad interna de la institución, y usan menos las que se refieren a la relación eficaz de la persona consigo misma y con el entorno (p. 1). 
Por otro lado, los fundamentos teóricos de artículo parte de tres conceptualizaciones relacionadas entre sí: la capacidad gerencial, la competencia y la habilidad. La capacidad gerencial se entiende como el conjunto de competencias profesionales y habilidades de las personas directivas que inteactúan en la persona para en el desarrollo de la gestión educativa.

La capacidad gerencial se caracteriza por establecer qué es lo que conocemos, dónde está el conocimiento y cómo puede aplicar y mejorar el bienestar de la organización escolar por medio de ella. El filósofo Amartya Sen, en su Enfoque de Capacidades, señaló, que para que una capacidad sea valiosa debe contemplar el bienestar y la libertad de las personas tanto a nivel individual como grupal; señaló Sen (1985):

La capacidad es el bienestar en términos de la habilidad de una persona para realizar actos valiosos, centrándose en el ser y el hacer, y que repercuten en el bienestar individual y grupal de la sociedad. En otras palabras, la capacidad de una persona es el conjunto de vectores de funcionamiento que reflejan la libertad de la persona para alcanzar aquello que valora generalmente determinado por las condiciones sociales, políticas y económicas del contexto (p. 5).

Entonces el elemento sustantivo de la capacidad es el funcionamiento y el enfoque de capacidades lo desarrolla explicando que la vida es un conjunto de funcionamientos que se encuentran interrelacionados y tienden a ser consistentes en estados y acciones. Es decir que los funcionamientos no son aislados, sino que están entrelazados para conformar un todo, además de ser permanentes en su estado.

Los funcionamientos se clasifican en dos tipos: los sencillos como, por ejemplo, la buena salud física y los complejos relacionados con la felicidad, la dignidad, la participación en comunidad y otros. La capacidad entendida como el bienestar para ser y hacer las actividades de la vida debe considerar los funcionamientos, ya que de estos dependerá la capacidad para el desarrollo personal o grupal, o por el contrario al deterioro de la capacidad debido al mal funcionamiento. La relación simbiótica entre la capacidad y el funcionamiento es bidireccional porque el funcionamiento alimenta a la capacidad y la capacidad juzga la función del funcionamiento. Por esta razón se puede decir que la capacidad es el conjunto de $n$ funcionamientos alcanzados.

Ahora bien, las personas directivas de instituciones educativas están llamadas a tomar conciencia de la concepción de capacidad en el tanto que el bienestar de una institución educativa va a depender de la habilidad de la persona directiva. Concretamente, la habilidad para hacer actos valiosos y significativos en la gestión educativa, centrándose en el ser (misión y visión institucional) y el hacer (desarrollo de proyectos, programas, actividades pedagógicas, entre otros), que van a repercutir en el bienestar individual y grupal de la comunidad educativa. Además, si la capacidad es el conjunto de $n$ funcionamientos, tanto profesionales como institucionales, para concretar acciones, se debe tener una clara comprensión sobre los funcionamientos individuales o personales de las personas directivas, lo cual implica conocerse a sí mismo tanto en sus funcionamientos sencillos (salud física, emocional, mental, etc.) como es su propio bienestar personal, así como de los funcionamientos complejos tales como las relaciones interpersonales, el trabajo en equipo; es decir, el bienestar institucional o grupal. Urquijo (2014) apunta lo siguiente:

La teoría de las capacidades propuesta por Sen no cae en el fetiche de los funcionamientos, se admite la importancia de los funcionamientos, pero se reconoce que según cada persona y los diferentes contextos en los que las personas se desarrollan, algunos funcionamientos adquieren mayor criterio de valoración que otros. Ahora bien, el conjunto de funcionamientos refleja la capacidad con que cuenta una persona para alcanzar aquello que valora (p. 71.)

Es importante mencionar que para potenciar la capacidad gerencial de las personas directivas escolares se debe contemplar el contexto, el desarrollo profesional, la realidad histórica de la institución, entre 
otros. Por tanto, para efectos de este estudio interesa ver los funcionamientos como las competencias necesarias que requieren las personas directivas para el buen desempeño de la capacidad gerencial. Ahora bien, Levy-Leboyer (2003) citado por Moya y Luengo y Asociación Proyecto Atlántida (2011) en la teoría y práctica de las competencias básicas arroja dos conclusiones del término competencia, a saber:

1. El término competencia se refiere al conjunto de acciones o decisiones que una persona o institución "puede" adoptar. En este sentido, "tener competencia" es poder decidir sobre algo.

2. El término competencia se refiere al modo en que una persona o institución utiliza sus posibilidades de decisión para adoptar "buenas" decisiones. En este sentido, "tener competencia" es sinónimo de saber (p. 31).

Ambos significados de competencia se ajustan al concepto de funcionamiento de la teoría de capacidades en el tanto que las competencias, al igual que los funcionamientos, permiten en primera instancia poder tomar decisiones; es decir, la libertad como condición necesaria del ser (Montuschi, 2008) y, en segunda competencia, saber cómo hacer. En una línea similar se puede encontrar el enfoque por competencias que retoma la UNESCO (2000) en la producción Competencias para la profesionalización de la gestión educativa, donde se conceptualiza competencia como:

(...) un conjunto integrado de conocimientos, capacidades, representaciones y comportamientos movilizados para resolver problemas profesionales. Ser competente implica poder realizar una actividad profesional, resolver problemas o desarrollar proyectos en tiempo oportuno movilizando integralmente un saber realizar, conjuntamente con los saberes conceptuales pertinentes y con capacidades diversas de acción y de relación, con el fin de obtener resultados de calidad. Implica conocer lo que se realiza, tener conciencia de sus consecuencias y capacidad de evaluar la acción. Supone capacidad para aprender, innovar y generar nuevos conocimientos. Otra característica de las competencias es su capacidad de transferibilidad hacia otras situaciones o procesos de acción. No es un saber válido exclusivamente en tareas específicas o rutinarias. La fortaleza de la formación basada en competencias reside en la posibilidad de ampliación de los saberes más allá de la simple ejecución de tareas delimitadas. En este sentido, ser competente supone la capacidad de ver, analizar e intervenir en un mundo complejo en el que se interrelacionan aspectos estrictamente técnicos con aspectos culturales, sociales, éticos, políticos y tecnológicos (p. 24).

En la conceptualización anterior, destaca la idea de competencia como la integración de habilidades personales, profesionales, saberes conceptuales y la experiencia de las personas directivas para el logro de los objetivos institucionales. Para la investigación, relacionar la conceptualización de competencia con el concepto de capacidad de Armatya Sen constituye una nueva visión de cómo integrar las competencias con capacidades como el bienestar, en términos de la habilidad de una persona para hacer actos valiosos, centrándose en el ser y hacer, y que repercuten en el bienestar individual y grupal de la sociedad (Urquijo A. 2014). Por consiguiente, la capacidad de las personas directivas radica en integrar las competencias para el logro del bienestar tanto profesional como institucional, desarrollando cada vez más sus capacidades individuales con las colectivas con el fin de atender los desafíos educativos que se viven en los centros y con las Juntas. Por otro lado, las competencias necesitan de una adecuada articulación entre ellas y para que esta articulación se logre se requiere de habilidades personales y profesional. Para Portillo (2017).

No existe una única definición sobre qué es una habilidad. Este término está siendo utilizado en los diferentes países sin tener un consenso. La definiciones dependen del lente desde el cual se mira: el trabajo, la comunicación, la tecnología, la sociedad, lo psicosocial, la política. Lo cierto es que la habilidad es un constructo social (Rigby y Sanchis, 2006) (p. 3). 
No obstante, para Grifin y Care (2014) una habilidad corresponde a las acciones que las personas pueden realizar para solventar determinada situación, por lo tanto las habilidades se desarrollan y pueden ser cambiantes, pues se ajustan de acuerdo con las exigencias del momento y del contexto. Esta articulación de habilidades para el desarrollo de competencias, son las que van a potenciar la capacidad gerencial de la persona directiva, aspecto central de este manuscrito.

La UNESCO (2000) identifica siete competencias necesarias para los responsables de la gestión educativa. Para efectos del estudio, se presenta un resumen de cada una con su respectiva conceptualización y procesos teóricos y prácticos en la tabla 1.

TABLA 1

Resumen de competencias para la gestión educativa según UNESCO

\section{Competencia / Conceptualización}

\section{LIDERAZGO}

El liderazgo puede definirse como el conjunto de procesos que orientan a las personas y a los equipos en una determinada dirección hacia el logro de la excelencia y el aprendizaje organizacional, primordialmente por medios no coercitivos (p. 9).

El liderazgo se vincula con la capacidad de generar procesos de sensibilización y convocatoria al trabajar en colaboración con otros, en el logro de los fines y los valores generalmente sepultados en el fárrago de las rutinas cotidianas (p. 9).

\section{COMUNICACIÓN}

La comunicación permite una doble apertura: hacia el entorno del sistema, tanto para emitir como para recibir comunicaciones de las familias, egresados, actores sociales (empresarios, sindicatos, etcétera). Hacia dentro del sistema, para fortalecer la integración con comunicaciones recíprocas en las que no sólo todos pueden hablar con todos, sino que además todos pueden intercambiar los roles de hablantes y oyenes (p. 12).

\section{DELEGACIÓN}

Delegar es otorgar a un colaborador -individual o grupal- de forma temporaria o permanente, la autoridad necesaria para tratar y decidir, con el encuadre explicitado y dentro de un ámbito preciso, haciéndolo/s responsable/s de los resultados de esa acción. Pero vale advertir que, si bien el delegatario es responsable ante el delegador, es siempre el responsable ante su superior (p.12).

\section{NEGOCIACIÓN}

La negociación puede ser entendida como el proceso dinámico en el cual dos o más actores en conflicto posible o manifiesto, o con intereses divergentes, entablan una comunicación para generar una solución aceptable de sus diferencias que se explicita en un compromiso (p. 16).

\section{Procesos teóricos y prácticos} para evidenciar la competencia
1. Inspirar la necesidad de generar transformaciones.

2. Generar una visión de futuro. Comunicar esa visión de futuro.

3. Promover el trabajo en equipos.

4. Actualizar el aprendizaje y acumular conocimiento.
1. Habilitar la comunicación, crucial en los modelos de gestión educativa.

2. Regular el manejo del tiempo en la comunicación, aspecto muy importante.

3. Permitir la interacción de las situaciones, competencias y necesidades.

4. Potenciar los procesos de cambio a partir de experiencias y recursos compartidos.

5. Vincular tanto a los actores internos como a los actores externos al sistema educativo.

1. Ampliar la autonomía y la toma de decisiones colegiadas.

2. Delegar para revalorizar.

3. Aumentar la eficacia.

4. Enfrentar la complejidad creciente.

5. Fortalecer las competencias colectivas.

6. Aumentar los niveles de responsabilidad, compromiso y pertenencia.
6. Proponer una metodología donde los conflictos pueden ser resueltos a través de un enfoque donde todos ganan por la misma asociación y se denomina postura de "ganador-ganador".

7. Analizar y comprender los intereses propios, los opuestos y los diferentes.

8. Instalar el diálogo y la confianza para hablar y escuchar.

9. Comprender que es un proceso para lograr acuerdos. 


\section{Competencia / Conceptualización}

\section{RESOLUCIÓN DE PROBLEMAS}

Es un método que permite no solamente resolver un problema puntual (lo cual hace su propia eficiencia), sino también deberá ser vista como una estrategia explícita que permite crear, adquirir y transferir nuevos conocimientos (p. 7).
Procesos teóricos y prácticos para evidenciar la competencia
1. Permitir la acción.

2. Brindar un enfoque global y sistémico.

3. Proponer para el aprendizaje permanente.

4. Posibilitar el mejoramiento continuo de las instituciones.

5. Desafiar las soluciones conocidas.

6. Proveer de una estrategia fundamental a los equipos de gestión y piloteo de sistemas complejos.

7. Articular las tareas inmediatas con las perspectivas de largo plazo.

8. Posibilitar el identificar soluciones diferentes.

\section{ANTICIPACIÓN}

La prospectiva puede entenderse como una mirada al futuro que se realiza para clarificar las acciones del presente. Michel Godet la define como un "panorama de los futuros posibles de un sistema destinado a clarificar las consecuencias de las acciones encaradas" (p. 5).

\section{TRABAJO EN EQUIPO}

El trabajo en equipo es una modalidad para articular las actividades laborales de un grupo humano en torno a un conjunto de fines, de metas y de resultados por alcanzar. El trabajo en equipo implica una interdependencia activa entre los integrantes de un grupo que comparten y asumen una misión de trabajo (p. 5).
1. Aplicar métodos de prospectiva.

1. Motivar la transformación de la cultura de trabajo.

2. Estimular la capacidad de trabajar en redes de colaboración.

3. Valoriza las reuniones de trabajo.

4. Articular el trabajo alrededor de proyectos.

5. Estimular la formación continua de los equipos de gestión.

Fuente: Adaptado de UNESCO (2000).

Tal y como se observa, los referentes teóricos presentan varias perspectivas que van del enfoque de las capacidades de Amartya Sen, al enfoque de competencias de la UNESCO; por cuanto el análisis de la capacidad gerencial de las direcciones debe ser estudiada y analizada bajo un enfoque interdisciplinario. Cabe indicar que todos los referentes permitirán aclarar y seleccionar la capacidad gerencial que se requiere para la administración de las personas directivas.

\section{MATERIALES Y MÉTODOS}

Bellido, Díaz, Morales y Pino (2009) señalan que la gestión educativa es exitosa si se dan capacidades gerenciales o de liderazgo en las personas directivas de los centros. De modo que para lograr esta situación se requiere que "todas las acciones que se implementen en el centro a nivel de gestión educativa, impliquen planificación, ejecución y control de las decisiones y acciones (p. 43)" desde diferentes ópticas en donde participan varios actores según sus funciones.

Así, para analizar las competencias que potencian la capacidad gerencial de las personas directivas de centros educativos para el trabajo efectivo con las Juntas se establecieron las siguientes etapas:

1. Identificación de las direcciones de centro educativo que han sido exitosas en la eficiencia y eficacia con las Juntas en cuatro direcciones regionales del Ministerio de Educación Pública donde el trabajo del directivo fuese proactivo y cumpliese con algunos criterios del Modelo de Excelencia de la Fundación Europea para la Gestión de la Calidad (EFQM por sus siglas en inglés);

2. Identificación teórica de capacidades gerenciales para la gestión educativa de modo que fuese una base para la investigación que se implementó (modelo de competencias de la UNESCO). 
Posterior a esto, se conjuntaron las competencias que potencian la capacidad gerencial de las personas directivas, paralelo al anterior proceso;

3. Selección de una muestra por conveniencia de cinco instituciones educativas en donde la persona directiva tuviese participación de la comunidad y Junta en la mejora de sus propias prácticas;

4. Análisis crítico de las situaciones, vinculadas con la práctica que tiene la persona directiva con la Junta.

5. Sistematización de las competencias que potencian la capacidad gerencial de las personas directivas y que optimicen la labor conjunta con las Juntas, a partir del análisis críticos y la aplicación de instrumentos.

Para llevar a cabo las etapas anteriores, por la naturaleza del objeto de estudio, se planteó una investigación cualitativa la cual, según Hernández, Fernández y Baptista (2014) "se enfoca en comprender los fenómenos, explorándolos desde la perspectiva de los participantes en un ambiente natural y en relación con su contexto (p. 358)".

Por tanto, una vez identificadas las competencias que potencian la capacidad gerencial y fueron conocidas por los participantes del estudio, son éstos sujetos quienes "(...) perciben y experimentan los fenómenos que los rodean, profundizando en sus puntos de vista, interpretaciones y significados (p. 358)", de modo que las personas directivas reflexionan, escudriñan, analizan y comprenden su realidad como agentes de cambio e intermediarios para el trabajo efectivo con la Junta, provocando que sean ellos mismos quienes prioricen y seleccionen, dentro del conjunto, las principales competencias gerenciales para lograr experiencias exitosas.

Ahora bien, la recopilación de información del proceso fue realizada mediante las técnicas de análisis de contenido, entrevistas y encuesta. Los instrumentos empleados son la matriz de análisis de contenido, la guía de entrevista semiestructurada y el cuestionario semiestructurado, respectivamente. Estos instrumentos responden a las tres grandes categorías de la investigación: competencias requeridas para potenciar la capacidad gerencial, la relación de las competencias detectadas de las personas directivas para la atención y solución de problemas, y los desafíos para el trabajo efectivo con la Junta.

Los instrumentos citados fueron validados mediante juicio de expertos y se incorporaron las sugerencias respectivas para la posterior aplicación. Los reactivos de la entrevista y el cuestionario consideran las competencias (y su jerarquía) que un directivo debe tener para trabajar con los miembros de Junta, cómo resolver problemas (situaciones y estrategias) en las prácticas con las Juntas y los desafíos para la mejora continua.

Los participantes fueron nueve personas directivas de Educación General Básica del Sistema Educativo Público Costarricense, provenientes de cuatro Regiones Educativas (San José Oeste, San José Central, Alajuela y Heredia), quienes se seleccionaron según criterios del Modelo de Excelencia de la Fundación Europea para la Gestión de la Calidad (EFQM). Modelo que, según Femenía-Millet (2011), "ofrece una expresión ordenada y sistemática para la gestión de las organizaciones, siendo un paradigma que incluye principios, procedimientos y valores y es considerado como una de las mejores estrategias de gestión de las organizaciones (p. 3)". 
Figura 1. Adaptación del Modelo de Excelencia de la Fundación Europea para la Gestión de la Calidad (EFQM) en contextos educativos



Fuente: Adaptación propia con base en Femenía-Miller (2011).

Con este procedimiento, los criterios de casos de personas directivas escolares con prácticas exitosas con Juntas fueron otorgados por el modelo según los agentes; es decir, los investigadores de este estudio, como parte de la investigación evidenciaron el liderazgo en la gestión del talento humano (docentes), la gestión del currículo (estudiantes) y los recursos para administrar de esos centros, pues conducían a un trabajo eficaz con la Junta y, por ende, a resultados de satisfacción e impacto en el servicio brindado. Tal como anota Urquijo (2014) "no importa lo que la persona tiene, sino lo que consigue realizar con lo que tiene (p. 69)", de ahí la importancia de que los sujetos de estudio se apoderen de la situación, sean partícipes de la investigación y se empoderen para generar el cambio. Por último, en cuanto a las consideraciones éticas del estudio, se trabajó mediante consentimientos informados para la obtención de la información por parte de los participantes, donde se les aseguró la confidencialidad del caso.

\section{RESULTADOS Y DISCUSIÓN}

En cuanto a los resultados de la investigación, se inicia con la primera categoría relacionada con la identificación de las competencias necesarias para la gestión educativa de las personas directivas y su trabajo con las Juntas. Se identifican cinco tipos de competencias prioritarias de nueve señaladas en la teoría presentada por la UNESCO (2000). Para estudiar el fenómeno se agrupan las cinco competencias prioritarias con sus competencias requeridas, tal como se muestra en la tabla 2. Las competencias prioritarias fueron identificadas por las personas directivas de acuerdo con el orden de importancia de su experiencia, esto fue determinado de manera cuantitativa en el instrumento creado para tal efecto y las competencias requeridas fueron señaladas en segundo orden de importancia. Las competencias, tal como se indicó, responden a situaciones teóricas y prácticas de las competencias, de acuerdo con la formulación teórica de la UNESCO (2000).

La primer competencia prioritaria que se identifica es el liderazgo, la cual orienta al directivo escolar hacia el logro de la calidad, con el fin de inspirar por medio de la misión y la visión institucional el espíritu de éxito. Para ello requiere de otras competencias requeridas tales como la comunicación, que permite la reflexión para el trabajo colaborativo y el compromiso de los miembros de las Juntas en la ejecución de actividades, tareas, programas, proyectos y otros; el trabajo en equipo, para potenciar los liderazgos de las personas miembros de las Juntas; la anticipación, que permite liderar que los planes de trabajo tengan prospectiva; la negociación, dirigida a un liderazgo compartido donde se dialoga e intercambian intereses. 
TABLA 2

Competencias y habilidades requeridas para la capacidad gerencial

\begin{tabular}{|c|c|c|c|}
\hline $\begin{array}{l}\text { Competencias } \\
\text { prioritarias } \\
\text { de la UNESCO }\end{array}$ & $\begin{array}{l}\text { Competencias } \\
\text { requeridas } \\
\text { de la UNESCO }\end{array}$ & $\begin{array}{l}\text { Habilidades manifestadas por las personas } \\
\text { directivas en la entrevista que evidencian } \\
\text { la competencia }\end{array}$ & $\begin{array}{l}\text { Frecuencia relativa de algunas } \\
\text { acciones afirmativas que evidencian } \\
\text { la competencia según información } \\
\text { del cuestionario }\end{array}$ \\
\hline Liderazgo & $\begin{array}{l}\text { Comunicación } \\
\text { Trabajo en equipo } \\
\text { Anticipación } \\
\text { Negociación }\end{array}$ & $\begin{array}{l}\text { Vivencia Común } \\
\text { Honestidad } \\
\text { Vocación } \\
\text { Visión para nuevos proyectos necesarios } \\
\text { para la institución } \\
\text { Responsabilidad } \\
\text { Compromiso } \\
\text { Inteligencia emocional }\end{array}$ & $\begin{array}{l}\text { Realiza las gestiones que le corresponden } \\
\text { ante diferentes entes, aunque impliquen } \\
\text { muchos trámites: } 100 \% \\
\text { El centro educativo se destaca por lograr } \\
\text { resultados positivos en diversas áreas: } \\
100 \%\end{array}$ \\
\hline Comunicación & $\begin{array}{l}\text { Trabajo en equipo } \\
\text { Resolución de } \\
\text { problemas }\end{array}$ & $\begin{array}{l}\text { Disposición } \\
\text { Conocimiento } \\
\text { Compromiso } \\
\text { Confianza } \\
\text { Empatía } \\
\text { Toma de decisiones } \\
\text { Inteligencia emocional } \\
\text { Conciencia plena } \\
\text { Escucha activa }\end{array}$ & $\begin{array}{l}\text { Comparte en las primeras reuniones la lí- } \\
\text { nea de trabajo: } 100 \% \\
\text { Favorece una comunicación clara con toda } \\
\text { la comunidad educativa: } 90 \% \\
\text { Se mantiene actualizado y dando a cono- } \\
\text { cer nuevas normativas que corresponden a } \\
\text { la Junta: } 100 \%\end{array}$ \\
\hline Negociación & $\begin{array}{l}\text { Comunicación } \\
\text { Trabajo en equipo } \\
\text { Liderazgo } \\
\text { Anticipación }\end{array}$ & $\begin{array}{l}\text { Toma de decisiones } \\
\text { Disposición } \\
\text { Creatividad } \\
\text { Honestidad } \\
\text { Compromiso }\end{array}$ & $\begin{array}{l}\text { Prepara, junto a otro miembro de Junta, la } \\
\text { agenda de proyectos por atender y en las } \\
\text { sesiones respectivas: } 100 \% \\
\text { Analiza las necesidades del centro educati- } \\
\text { vo con los miembros de la Junta: } 100 \%\end{array}$ \\
\hline $\begin{array}{l}\text { Trabajo en } \\
\text { Equipo }\end{array}$ & $\begin{array}{l}\text { Comunicación } \\
\text { Negociación } \\
\text { Liderazgo } \\
\text { Anticipación }\end{array}$ & $\begin{array}{l}\text { Visión global y anticipada } \\
\text { Empatía } \\
\text { Creatividad } \\
\text { Objetivo-meta en común para trabajar en conjunto } \\
\text { Compromiso }\end{array}$ & $\begin{array}{l}\text { Permite y colabora para que los miembros } \\
\text { de las Juntas se empoderen en la compren- } \\
\text { sión del funcionamiento de la misma: } 70 \% \\
\text { Logra resolver los problemas de manera } \\
\text { colaborativa con otros miembros de Junta: } \\
90 \%\end{array}$ \\
\hline Delegación & $\begin{array}{l}\text { Liderazgo } \\
\text { Escucha activa } \\
\text { Negociación }\end{array}$ & $\begin{array}{l}\text { Conocimiento } \\
\text { Escucha activa } \\
\text { Disposición } \\
\text { Conocimiento }\end{array}$ & $\begin{array}{l}\text { Capacita a los miembros de la comunidad } \\
\text { educativa: } 100 \%\end{array}$ \\
\hline
\end{tabular}

Fuente: Elaboración propia con base en los resultados de la entrevista y el cuestionario semiestructurado aplicado a las personas directivas (2020) y la propuesta de competencias de la UNESCO (2000).

Los resultados muestran que la segunda competencia prioritaria es la comunicación. Las personas directivas señalan que la comunicación es uno de los aspectos vitales para la coordinación y el trabajo con la Juntas. Siendo la escucha activa una de las habilidades que permiten generar compromiso, disposición, empatía y confianza para la toma de decisiones orientadas a fortalecer y ser efectivos en la concreción de proyectos, actividades, programas u otros. 
Un tercer resultado identifica la negociación como otra competencia prioritaria, por cuanto las presiones, tanto de las personas directivas como los miembros de las Juntas, pueden presentar, y de hecho lo hacen, necesidades e intereses divergentes que generan conflictos. La negociación es una competencia para que las partes se reúnan y lleguen a acuerdos significativos y viables para el bienestar de la comunidad estudiantil.

El trabajo en equipo es la cuarta competencia que identifican las personas directivas para el trabajo efectivo con las Juntas. La planificación de acciones concretas para el desarrollo de proyectos en los centros educativos debe ser una labor conjunta de la comunidad educativa en torno al logro de metas y resultados institucionales. El trabajo en equipo entre la persona directiva y la Junta permite contar con una visión global y anticipada de los objetivos y metas comunes. Genera, por consiguiente, el compromiso, la empatía y la creatividad de todos sus miembros.

El quinto resultado identifica la delegación como competencia prioritaria. Las personas directivas consideran que un líder debe saber tomar decisiones, pero sobre todo debe saber delegar funciones tanto de forma individual como grupal. Si bien es cierto que las personas directivas solo tienen derecho a voz y no a voto dentro de la Junta, ésta es la responsable de los resultados de sus acciones. Por consiguiente, la delegación permite motivar y generar pertenencia, desarrolla el juicio discrecional e incrementa los niveles de confianza.

De acuerdo con la segunda categoría establecida: relación de las competencias detectadas de las personas directivas para la atención y solución de problemas, las personas directivas entrevistadas la señalan como clave para un trabajo efectivo con la Junta y, en general, con la comunidad educativa, dado que potencia el logro de los objetivos comunes. Ahora bien, si se contrasta con la teoría propuesta por UNESCO (2000) se evidencia que las personas directivas en efecto manifiestan la competencia. Nótese la tabla 3.

TABLA 3

Evidencias de la resolución de problemas en las personas directivas

\section{Procesos teóricos y prácticos vinculados con la competencia "Resolución de problemas" según UNESCO (2000).}

Emplea una metodología para la acción.

Presenta un enfoque global y sistémico.

\section{Escucha activa.}

Posibilita el mejoramiento continuo de las instituciones

Emplea una estrategia que desafía las soluciones conocidas.

\section{Acciones realizadas por las personas directivas que evidencia la competencia "Resolución de problemas" según entrevista}

Fomenta el liderazgo democrático.

Oaniza con la Junta los procesos macro de administración del centro, escuchándolos, considerándolos, siguiéndoles para una mejor toma de decisiones.

Visita el hogar de los estudiantes.

Se comunica constantemente con diversos actores educativos. Negociación con miembros de Junta para logro de actividades.

Propicia el empoderamiento de la comunidad y sentido de pertenencia con el centro educativo.

Promueve alianzas con organizaciones privadas.

Hace partícipes a los profesores de los problemas para que, de manera corresponsable, se aúnen esfuerzos de solución.

\section{Frecuencia relativa de acciones afirmativas para atender los problemas según cuestionario}

Colabora para que diversas personas se postulen como miembro de la Junta: $90 \%$

Prepara, junto a otro miembro de Junta, la agenda de proyectos por atender y en las sesiones respectivas: $100 \%$

Corrige errores que surgen en la gestión de la Junta desde el primer momento que aparecen: $100 \%$

El centro educativo se destaca por lograr resultados positivos den diversas áreas: $100 \%$

Logra terminar con éxito en el tiempo previsto los proyectos que se plantean con la Junta: $87 \%$ 


\section{Procesos teóricos y prácticos vinculados con la competencia \\ "Resolución de problemas" según UNESCO (2000).}

Promueve el trabajo en equipos de gestión.

\section{Acciones realizadas por las personas \\ directivas que evidencia la competencia \\ "Resolución de problemas" \\ según entrevista}

Da seguimiento a los procesos administrativos.

Promueve, en la medida de lo posible, la continuidad del personal docente.

Realiza actividades de diversa índoles, de la mano con la Junta, para el beneficio de la comunidad educativa.

Identifica los roles de miembros de Junta y docentes para mejorar procesos.

Crea una cultura de gestión.

Articula las tareas inmediatas con las perspectivas de largo plazo.

Realiza de diagnóstico situacional del centro educativo.

Formula un proyecto quinquenal con la municipalidad para atención de necesidades infraestructurales.

Busca recursos propios para la solución de problemas cotidianos, por ejemplo, baterías sanitarias, gimnasio, comedor, pupitres, entre otros.

Propone una oferta educativa diferente y atractiva, previa autorización del MEP.

Identifica soluciones diferentes.

\section{Frecuencia relativa \\ de acciones afirmativas para atender los problemas según cuestionario}

Logra resolver los problemas de manera colaborativa con otros miembros de Junta: $90 \%$

Fuente: Elaboración propia con base en los resultados de la entrevista y el cuestionario aplicado a las personas directivas (2020) y la propuesta de competencias de la UNESCO (2000).

Tal como se muestra en la tabla 3, las personas directivas de los centros educativos evidencian la competencia gerencial de resolución de problemas al promover acciones para solventar las diversas situaciones, por ejemplo, comunicación efectiva, la planificación estratégica, el asumir el problema de forma oportuna, la integración de equipos para la solución, la proactividad, entre otros. Al respecto, es importante, tal como plantea UNESCO (2000), que además de resolver el problema, construyen nuevos conocimientos como estrategia de permanencia en el centro educativo (p. 7).

De acuerdo con García (2000), las personas directivas son eficaces en la resolución de problemas si son organizados, se vinculan con la comunidad y son ejemplos de un liderazgo auténtico, aspectos que, como queda expuesto hasta el momento, se visualizan en los casos analizados. Indica el autor que "estos directores tienen habilidades de gestión, no sólo con el personal adscrito a su centro, sino con otras personalidades de la comunidad (p. 5)". Good y Brophy (1986) mencionan que "los directores eficaces crean un sentimiento de comunidad, con valores, cultura y objetivos compartidos (p. 45)", por ende, en este caso, demuestran capacidades gerenciales para el trabajo efectivo con las Juntas en el contexto educativo costarricense.

Por último, relacionado con la tercera categoría de la investigación (desafíos para el trabajo efectivo con la Junta), es necesario indicar que el proceso de dirigir y/o gestionar un centro educativo, ya de por sí mismo es un desafío, razón por la cual, el presentar competencias no es solo requerido en el perfil profesional de las personas directivas, sino necesario para dar respuesta a trabajos efectivos. La cultura de trabajo en equipo, la comunicación constante, el confiar en la delegación y la disposición a la negociación son procesos clave en el rol y liderazgo de la persona directiva del centro.

De este modo, el éxito de gerenciar un centro educativo dependerá, tal como lo propone UNESCO (2000), de las competencias antes señaladas e identificadas por las personas directivas en las sesiones de trabajo de esta investigación. De acuerdo con Sepúlveda y Aparicio (2017): 
En la actualidad, la gestión de una escuela requiere una apertura hacia un trabajo más colaborativo donde se establezcan metas en común y todos los profesionales de la educación estén dispuestos a probar nuevos métodos y aprender de experiencias ya desarrolladas. (p. 13).

Nótese, que las competencias identificadas en la investigación coinciden y reafirman la teoría seleccionada para la investigación, de modo que la cultura organizacional del centro educativo (incluyendo la Junta, como órgano colegiado), debe ser motivada, empoderada y resiliente, ver tabla 4.

TABLA 4

Desafíos para el desarrollo de la capacidad gerencial y trabajo efectivo con las Juntas

Desafíos para el trabajo efectivo con las Juntas

desde la perspectiva de las personas directivas

Gestionar oportunamente los trámites por parte de la Junta

Crear una visión compartida de proyecto educativo.

Ver las oportunidades de mejora, en lugar de lo negativo.

Empoderar a la Junta.

Buscar recursos didácticos para la mejora del centro.

Negociar con la Junta los proyectos prioritarios.

Dar respuestas oportunidades a situaciones donde se sabe que no

se puede hacer mucho.

Comunicación asertiva entre todos los miembros de la comunidad

educativa.

\section{Frecuencia de acciones afirmativas para atender los desafíos enfrentados desde la perspectiva de las personas directivas}

Permite y colabora para que los miembros de la Junta se empoderen en la comprensión del funcionamiento de la misma: 70\%

Capacita a los miembros de la comunidad educativa: 100\%

Comparte en las primeras reuniones la línea de trabajo: 100\%

Favorece una comunicación clara con toda la comunidad educativa: $90 \%$

Brinda seguimientos periódicos a los acuerdos de la Junta: $100 \%$

Fuente: Elaboración propia con base en los resultados de la entrevista y el cuestionario aplicado a las personas directivas (2020).

Tal como se anota en la tabla 4, las acciones afirmativas para atender los desafíos pueden ser una muestra clara del desarrollo de la competencia principal, la competencias requerida y de la habilidad para el desarrollo de la capacidad gerencial, esto por cuanto evidencian posibilidades de solución de manera comunicativa, negociadora, buscando el trabajo en equipo, siendo así requerido el liderazgo y la delegación. No obstante, llama la atención la acción "Permite y colabora para que los miembros de las Juntas se empoderen en la comprensión del funcionamiento de la misma", por cuanto solo 70\% de las personas directivas participantes en la investigación la realizan, sugiere así que se debe fortalecer la competencia principal: colaboración.

\section{CONCLUSIONES}

Con respecto al objetivo primero de este estudio, se logran identificar cinco competencias principales, a saber: liderazgo, comunicación, negociación, trabajo en equipo y delegación, las cuales potencian la capacidad gerencial de las personas directivas hacia el trabajo efectivo con las Juntas. Cada una de estas competencias es fortalecida por otras competencias requeridas y habilidades que se evidencia con la metodología de investigación aplicada para el estudio, tal y como se muestra en la tabla 2. Cabe indicar que las cinco competencias principales, según los resultados, se retroalimentan interdependientemente, lo que permite el desarrollo de la capacidad gerencial de forma global de la persona directiva para el trabajo con la Junta.

El buen funcionamiento de cada una de las competencias y su articulación permiten el consolidar la capacidad, tal y como la define en la Teoría de las capacidades Armatya Sen: "la capacidad como el bienestar en términos de la habilidad de una persona para hacer actos valiosos" (Urquijo, 2014, p. 63). El cambio 
y el trabajo efectivo con las Juntas va a depender de la capacidad gerencial de la persona directiva y, por lo tanto, las acciones y toma de decisiones deben estar orientadas a ser valiosas para la comunidad educativa donde pertenece.

En relación con la categoría "relación de las competencias detectadas de las personas directivas para la atención y solución de problemas", es importante acotar que las realidades educativas del país son muy diferentes, complejas, multicausales, singulares y cambiantes. Sin embargo, en los casos estudiados en esta investigación se evidencia una serie de acciones como la comunicación efectiva, la planificación estratégica, el asumir el problema de forma oportuna, la integración de equipos para la solución, la proactividad, entre otros que, desde la teoría base, son esenciales para lograr una competencia gerencial. La forma en que se aborda, las particularidades y la naturaleza del problema en sí, dependen de un contexto, sin embargo, las personas directivas involucradas en la investigación reconocen desde su propia práctica una constante en la gestión directiva que puede ser replicable en muchos contextos y con otros homólogos. De este modo, se logra cumplir el objetivo pues queda claro que existe una relación de las competencias estudiadas de las personas directivas para la atención y solución de problemas, según lo manifestado en las tablas 2 y 3.

Por otra parte, el tercer objetivo vinculado con la categoría de desafíos, resultó que aunque hay un predominio de las competencias principales y requeridas, es necesario promover la colaboración con las Juntas para gestar el cambio. Esto es importante pues no todos las personas directivas tienden a realizarlo; algunas de las razones que esgrimen se vinculan con posiciones autoritarias de miembros de Juntas, poca preparación académica de los mismos, lo cual incide en un papel más activo y preponderante de la persona directiva por encima de la persona presidente de Junta, entre otros; a pesar de ello, los resultados del cuestionario aplicado evidencian la presencia de las habilidades mostradas en la tabla 2.

En síntesis, las competencias prioritarias, identificadas por las personas directivas para el trabajo efectivo con las Juntas versan en el liderazgo, la comunicación, la negociación, el trabajo en equipo y la delegación. En este sentido, esta investigación es un primer acercamiento para analizar las competencias que potencian la capacidad gerencial de quienes lideran centros educativos públicos en Costa Rica, para que realicen un cambios en sus entornos, por lo que se constituye en una base para futuros proyectos con el fin de incidir hacia un nuevo paradigma de gestión educativa con las juntas de educación y juntas administrativas y, sobre los requerimientos de formación inicial que deben considerarse para estos profesionales. Por lo anterior, es recomendable que en la educación superior los planes de estudio incluyan elementos de formación en estas competencias y otras, pues es necesario que en el marco de una política educativa denominada La persona: centro del proceso educativo y sujeto transformador de la sociedad (MEP, 2016, p. 1) las personas directivas fortalezcan su capacidad gerencial para la adecuada gestión escolar, pero también, requiere revista por parte del Ministerio de Educación Pública de Costa Rica, como mayor empleador de las personas directivas de educación para el fortalecimiento, mediante programas de desarrollo profesional continuo, de las competencias que potencien esa capacidad gerencial del director y la directora de centro educativo. 


\section{REFERENCIAS}

Bellido, A. C., Díaz Donado, J., F. Morales Ríos y Pino de Ochoa, L. (2009). Gestión y Supervisión en el Centro de Educación Básica (Vol. 36). San José Costa Rica: Coordinación Educativa y Cultural Centroamericana. Recuperado de http://www.ceducar.info/ceducar/recursos/biblioteca-virtual/ volumen36.pdf

Femenía-Millet, O. (2011). Indicadores que mejoran la calidad en los centros educativos. Ponencia presentada en Jornada Redes 2011 - Universidad Católica de Valencia. Recuperado de https://web. ua.es/en/ice/jornadas-redes-2011/documentos/proposals/182357.pdf

García, J. (2000). ¿Cuáles son las características del liderazgo del director y del supervisor de educación básica efectivo? Una revisión de la literatura internacional y la investigación generada en México. En: Psicología educativa: programas y desafíos en educación básica, pp. 109-124. México:UPN.

Gobierno de Costa Rica (2014). Decreto 38249 - MEP. Reglamento General de Juntas. Recuperado de http://www.pgrweb.go.cr/scij/Busqueda/Normativa/Normas/nrm_texto_completo.aspx?param $1=$ NRTC\&nValor1=1\&nValor2=76782\&nValor3=95968\&strTipM=TC

Good, T. y Brophy, J. (1986). School effects. En: Wittrock, M. (Ed.). Handbook of research on teaching, pp. 570-602. New York: MacMillan.

Griffin, P. y Care, E. (2014). Developing learner collaborative problem solving skills [Desarrollando habilidades de resolución de problemas en estudiantes]. Recuperado de https://sodas.ugdome.lt/ bylos/GENERAL/8af7dd98-d82c-4d81-90ed-7f912c0dfcf0.docx

Hernández, R., Fernández, C., Baptista, P. (2014). Metodología de la Investigación. Sexta Edición. México: Mc Graw Hill.

Ministerio de Educación Pública (2016). Política educativa: La persona: centro del proceso educativo y sujeto transformador de la sociedad. Recuperado de https://www.mep.go.cr/sites/default/files/ page/adjuntos/politicaeducativa.pdf

Montuschi, L (2008). La libertad, el libre albedrío y el enfoque de las capacidades: El desarrollo humano como libertad. Universidad del CEMA, Buenos Aires, Argentina. Recuperado de: https://ucema. edu.ar/publicaciones/download/documentos/372.pdf

Moya, J; Luengo, F. y Asociación Proyecto Atlántida (2011). Teoría y práctica de las competencias básicas. GRAO. España. Recuperado de: https://books.google.co.cr/books?id=yswOC5wXeGQC\&printsec $=$ frontcover\&hl=es\&source=gbs_ge_summary_r\&cad $=0 \# v=$ onepage $\& q \& f=f a l s e$

Patrón-Cortés, R. (2016). Competencias de directores académicos en instituciones de educación superior: Un estudio en el sureste de México. En Gestión de la Educación Vol. 6, N² 2, [1-18]. DOI: http://dx.doi.org/10.15517/rge.v1i2.25479

Portillo, M. (2017). Educación por habilidades: Perspectivas y retos para el sistema educativo. Revista de Educación. 2(41). DOI: http://dx.doi.org/10.15517/revedu.v41i2.21719

Pozner, P. (2007). El directivo docente como líder de la gestión educativa. Recuperado de 186.113.12.182 > catalogo > dlfileEl Directivo Docente como Líder de la Gestión Educativa

Sen, A. (1985). Commodities and Capabilities. Amsterdam: North-Holland. (Reimpreso en Delhi, Oxford University Press, 1999). Recuperado de: https://www2.uned.es/dpto_log/jalvarez/publicaciones/ sen2001.pdf

Sen, A. (1992). Inequality Reexamined. En A. Bravo y P. Schwartz (2000). Nuevo examen de la desigualdad. Madrid: Alianza. Recuperado de: http://revecap.com/revista/numeros/06/pdf/bandres.pdf

Sepúlveda, F. y Aparicio, C. (2017). El desafío de los directores de escuelas chilenas: Liderando a partir de un enfoque instruccional hacia un enfoque distribuido. Disponible en https://doi.org/10.15517/rge. v7i2.30599 
Suárez, D. (2015). Liderazgo Gerencial para la Resolución de Problemas. Recuperado de https://repository.unimilitar.edu.co/bitstream/handle/10654/6966/Disney\%20Suarez\%20Rodriguez\%202015. pdf;jsessionid=42837B4B475128A0DE9CEA8C0FF4E3C4?sequence $=1$

Torres, N. y Castro, O. (2012). Capacitación y actualización de las juntas de educación en escuelas indígenas en zonas rurales: Una manera de fortalecer la gestión educativa local. Revista Electrónica Educare, 16(3), 105-114. Recuperado de http://www.revistas.una.ac.cr/index.php/EDUCARE/issue/ current

UNESCO (2000) Competencias para la profesionalización de la gestión educativa: diez módulos destinados a los responsables de los procesos de transformación educativa. IIPE. Buenos Aires, Argentina. Recuperado de: https://unesdoc.unesco.org/ark:/48223/pf0000159155

Urquijo, M. (2014). La teoría de las capacidades en Amartya Sen. En Edetania: estudios y propuestas socio-educativas. №. 46. Recuperado de https://dialnet.unirioja.es/servlet/articulo?codigo $=5010857$

Para citar este documento:

Chen-Quesada, E. y Ruiz-Chaves, W. (2020). Competencias que potencian la capacidad gerencial en las personas directivas para el trabajo efectivo con las Juntas Administrativas y de Educación en el sistema educativo costarricense. Revista Innovaciones Educativas. 22(32), 36-50. DOI: https://doi.org/10.22458/ie.v22i32.2964 